\title{
A Mixture Peaks over Threshold Approach for Predicting Extreme Bridge Traffic Load Effects
}

\author{
Xiao-Yi Zhou ${ }^{1}$, Franziska Schmidt ${ }^{2}$, François Toutlemonde ${ }^{3}$ and Bernard Jacob ${ }^{4}$
}

\footnotetext{
${ }^{1}$ Formerly, Ph.D. student, Materials and Structures Department, IFSTTAR (French Institute of Science and Technology for Transport, Development and Networks); Université ParisEst, France; Currently, Research associate, Ph.D., School of civil engineering and geosciences, Newcastle University, United Kingdom.

${ }^{2}$ Research engineer, Ph.D., Materials and Structures Department, IFSTTAR; Université Paris-Est, France.

${ }^{3}$ Chief Scientist, Materials and Structures Department, IFSTTAR; Université Paris-Est, France.

${ }^{4}$ Senior engineer and Scientific delegate, IFSTTAR; Université Paris-Est, France.
} 
effects. Results show that the proposed approach is efficient to conduct extreme value analysis for data having mixture probability distribution function.

Keywords: Traffic load effects; Peaks-over-threshold; Mixture peaks-over-threshold; Bridge; Extreme value; Generalized Pareto distribution

\section{INTRODUCTION}

Assessing the condition of existing bridges is of increasing concern in bridge management as more and more bridges step into their ageing stage worldwide, and a deteriorated bridge raises a risk to safety and welfare loss for the users. Although extensive efforts have been devoted to elaborate load-carrying capacity models, the role of traffic loading in existing bridge structures has increasingly received attention in recent years as potential benefits have been revealed in terms of optimally allocating the limited maintenance and management budgets (COST 345 2002; Frangopol et al. 2008; Fu and You 2009; Li et al. 2012). In addition, the growth of traffic has been reported in recent years worldwide: for instance in Europe the road freight transport has increased by 35\% between 1995 and 2010. This has led the regulators introducing truck weight limit regulations and allowing the introduction of higher and longer vehicles in some member states, such as Scandinavia. These changes may have aggressive impacts on bridge structures in terms of maximum load and load effect, fatigue damage, probability of failure and etc. (Desrosiers and Grillo 1973; Ghosn and Moses 2000; Righiniotis 2006; Gindy and Nassif 2007; Tong et al. 2008; Fu et al. 2011; Zhou et al. 2014; O'Brien et al. 2014). The topic of multi-hazard analysis combines traffic loading with seismic or wind loading (Cai and Chen 2004; Zhu and Frangopol 2012; Ghosh et al. 2013). Therefore, an accurate prediction of the extreme traffic load effects on bridges is desired, especially for evaluating existing bridge structures.

Indeed the estimation of a high quantile or tail distribution is not an easy task, 
making inference about the extremal behaviour, in a domain where the samples only contain a very small amount of data. Moreover, extrapolation beyond the range of the data is necessary to know something about areas where there are no observation at all (Leadbetter et al. 1983; Coles 2001; de Haan and Ferreira 2006). This issue belongs to extreme value statistics, which has been extensively developed in the last 60 years, although it can be tracked back to the early 20th century. Extreme value predictive techniques have been used in many disciplines including structural engineering, and extensive research has been conducted in recent decades on bridge traffic load effects. The methods in the literature on extreme traffic load effects on bridges can be broadly classified into two major categories: (1) tail distribution methods, and (2) periodic maxima methods.

The primary objective of the first category of methods is to find the underlying distribution of bridge traffic load effects, then the maximum value distribution can be easily computed by raising the distribution to a certain power (Coles 2001). Using Normal distribution (Nowak 1993; Sivakumar et al. 2011), Gumbel distribution (Cooper 1997; Fu and You 2009), Weibull distribution (O'Brien et al. 1995) to bridge traffic load effects belongs to this category of method. In addition, the Rice formula based level-crossing method adopted in (Cremona 2001) can also be classified into the first category as the mathematical assumption implies that the traffic load effect is normally distributed.

The second category of methods aim at fitting a series of local maxima, taken from successive independent samples of observations over a given time period, to a standard extreme value distribution. Then the extreme characteristic values (or values with a given return period) for expected probabilities of exceedance can be computed. Fitting daily or yearly maxima to Weibull distribution (Bailey and Bez 1999), or to Gumbel distribution (Fu and You 2009) and to generalized extreme value distribution (Messervey et al. 2010; Park and Sohn 2006; Enright 
et al. 2013) belongs to this category. A comprehensive review and quantitative comparison of the prediction methods of extreme traffic load effects on bridges can be found in (O'Brien et al. 2015).

It has been widely accepted in the extreme value statistics research community that the generalized Pareto distribution (GPD) based peaks-over-threshold approach (POT) is as effective as generalized extreme value distribution (GEV) based block-maxima method (BM) to estimate extreme value. However, the use of POT approach has seldom been reported in bridge traffic load effects, although the POT approach has significant advantages. Many papers in other disciplines have proved that it may provide more accurate estimates than the BM method in modelling extreme values (Madsen et al. 1997; O'Brien et al. 2015). Moreover its mathematical form leads to very simple formulation.

Most of the previous works assume that bridge traffic load effects are identically and independently distributed (iid), which is a main condition to apply the extreme value theory (Coles 2001). However, it has been shown that bridge traffic load effects are induced by different types of loading events, depending on the number of trucks being simultaneously on the bridge deck. Thus the periodic maximum (usually daily maximum) used in the estimation may not come from the same type of distribution, which does not comply with the iid assumption (Harman and Davenport 1979). Desrosiers and Grillo (1973) stated that the multiple presence of trucks depends significantly on the bridge length, truck speed and traffic volume based on field data collected from several highway locations (Connecticut Route 5, I-91 at the Depot Hill Road, and I-91 at the Connecticut Route 68). These findings have been confirmed in (Gindy and Nassif 2007) with recent traffic data collected from 25 WIM sites in New Jersey between 1993 and 2003. Moreover, Messervey et al. (2010) states that the periodic maxima usually do not come from a single distribution as the number of events varies day by day. 
It is possible to select an optimal periodic length (Messervey et al. 2010), but it may waste data because of the reduced number of extremes used from these data. Another solution by (Caprani et al. 2008) named composite statistic distribution method accounts for the variation of loading distribution based on block maxima method and models extreme load effects from the same type of loading event.

In order to address the non-identically distributed traffic load effects, a novel extreme value analysis method has been proposed. The proposed method is based on the conventional peaks-over-threshold method (CPOT), which relies on the generalized Pareto distribution. The principle is to classify the traffic load effects by types of loading event. Then the CPOT is used to derive the upper tail of load effect distribution for each loading event category with generalized Pareto distribution. Finally the upper tail distribution is the weighed average of the upper tail distributions by loading event.

In the following sections, the mathematical background and the details of derivation of the novel method are presented. Numerical studies, including a theoretical example and a real traffic load effect example, are conducted to illustrate the capacity of the proposed method, and its performance is assessed by comparing with the conventional methods and the recently developed composite statistic distribution method (Caprani et al. 2008).

\section{METHODOLOGY}

\section{The generalized Pareto distribution and Peaks-over-Thresholds ap-} proach

Let $X_{1}, \ldots, X_{n}$ be a sequence of independently and identically distributed random variables with distribution function $F$. When the value taken by $X_{i}$ exceeds some high threshold $u$, this value can be treated as an extreme event. The behavior of those extremes can be described by the conditional distribution 
function of the excesses, $x=X-u$, over the threshold $u$ :

$$
F_{u}(x)=\operatorname{Pr}\{X-u \leq x \mid X>u\}=\frac{F(x+u)-F(u)}{1-F(u)},
$$

for $0 \leq x<x_{0}-u$.

The Balkema-de Haan-Pickands theorem (Balkema and de Haan 1974; Pickands III 1975) states that, for a certain class of distributions, the generalized Pareto distribution (GPD) is the limiting distribution for the distribution of the excesses, as the threshold tends to the right endpoint. The distribution function of GPD is usually expressed as:

$$
H(x ; \xi, \sigma)= \begin{cases}1-\left[1+\xi\left(\frac{x-u}{\sigma}\right)\right]^{-1 / \xi} & \xi \neq 0, \\ 1-\exp \left(-\frac{x-u}{\sigma}\right) & \xi=0,\end{cases}
$$

where $u$ is the threshold value, $\sigma>0$, and the support is $x \geq 0$ when $\xi \geq 0$ and $0 \leq x-\sigma / \xi$. The GPD comprises three known distribution types, depending on the value of parameter $\xi$. When $\xi>0$, the function is equivalent to a reparametrized version of the usual Pareto distribution; if $\xi<0$, the distribution is called a type II Pareto distribution; $\xi=0$ gives the exponential distribution.

According to Eqs. (1) and (2), the distribution function $F(x)$ can thus be expressed as:

$$
F(x)=\left(1-\varsigma_{u}\right)+\varsigma_{u} H(x ; \xi, \sigma, u),
$$

where $\varsigma_{u}=\operatorname{Pr}\{X>u \mid X \geq 0\}=1-F(u)$ represents the survival function, while $F_{u}(x)$ is the cumulative distribution function (CDF) of $x>u$ only.

The quantile $x_{m}$ that is exceeded on average once every $m$ observations is the 
solution of:

$$
x_{m}= \begin{cases}u+\frac{\sigma}{\xi}\left[\left(m \varsigma_{u}\right)^{\xi}-1\right] & \xi \neq 0 \\ u+\sigma \log (m \varsigma) & \xi=0\end{cases}
$$

provided $m$ is sufficiently large to ensure that $x_{m}>u$.

\section{Derivation of the mixture Peaks-over-Thresholds method}

Now, let $X_{1}, \cdots, X_{n}$ be a sequence of independently but non-identically distributed random variables with distribution function $F$, which is a mixture distribution consisting of $m$ components, expressed as:

$$
F(x)=\sum_{j=1}^{m} F_{j}(x) \cdot \varphi_{j}
$$

where the $j$-th component (distribution function of the $j$-th sub-population) $F_{j}$ belongs to the domain of maximum attraction, and $\varphi_{j}$ is the weight of $X$ belonging to the $j$-th sub-population, with $\sum_{j=1}^{m} \varphi_{j}=1$. Straightforwardly, the survivor function is expressed as:

$$
\bar{F}(x)=1-F(x)=\sum_{j=1}^{m}\left[1-F_{j}(x)\right] \varphi_{j} .
$$

Assume that for a given threshold $u_{j}$ the exceedances of $j$-th component could be reliably described by a generalized Pareto distribution, from Eq.(3) the survivor function of the $j$-th component can be formulated:

$$
1-F_{j}(x) \equiv\left[1-H_{j}\left(x-u_{j}\right)\right]\left[1-F_{j}\left(u_{j}\right)\right]
$$

1 Substituting Eq.(7) into Eq.(6), the survivor function of the mixture distribution 
can be expressed as:

$$
\bar{F}(x)=\sum_{j=1}^{n_{t}}\left[1-H_{j}\left(x-u_{j}\right)\right]\left[1-F_{j}\left(u_{j}\right)\right] \varphi_{j} .
$$

Therefore, the tail of the mixture distribution can be represented by:

$$
F(x)=1-\bar{F}(x)=1-\sum_{j=1}^{n_{t}}\left[1-H_{j}\left(x-u_{j}\right)\right]\left[1-F_{j}\left(u_{j}\right)\right] \varphi_{j} .
$$

54 obt 6 Sati satisfies the following equation:

$$
\left[1-F\left(\hat{x}_{m}\right)\right]-\frac{1}{m} \leq \epsilon
$$

with $\epsilon$ as a given small value.

\section{Approach for threshold selection in the use of Mixture Peaks-Over-} Thresholds method

In the application of the MPOT method, an essential step is to select an appropriate threshold $u_{j}$ for each component of the mixture models of load effects to which the asymptotic GPD is approximated. The threshold selection requires consideration of the trade-off between bias and variance: a too high threshold reduces the number of exceedances and thus increases the estimated variance, whereas a low threshold can reduce the estimated variance but increase the bias (Scarrott and MacDonald 2012). Graphical diagnosis approaches, e.g. the mean residual life plot, are commonly used for such a selection, but they require the practitioner to have substantial expertise and can be rather subjective. Moreover, they may be time-consuming if there are many thresholds to be selected. 
Hence, graphical diagnosis approaches are not fully suitable for our problem. Automatic threshold selection approach with appropriate measure is preferable to avoid subjective judgement and to apply the proposed approach efficiently as several thresholds are needed to be selected in the MPOT method. Several types of automatic threshold selection rules exist. The simplest ones are the fix number rules such as the upper $10 \%$ rule, the square root rule $k=\sqrt{n}$ or its modification $k=n^{2 / 3} / \log [\log (n)]$, but they are usually lacking of theoretical background.

Therefore, we adopt the automatic method based on goodness-of-fit test statistics. The Anderson-Darling (AD) and Cramer - von Mises (CM) test proposed by Choulakian and Stephens (2001) to examine the goodness-of-fit for GPD have been adopted:

$$
\begin{aligned}
W_{n}^{2} & =\frac{1}{12 n}+\sum_{i=1}^{n}\left(z_{i}-\frac{1-1 / 2}{n}\right)^{2} \quad \text { for CM test, } \\
A_{n}^{2} & =-n-\frac{1}{n} \sum_{i=1}^{n}(2 i-1)\left\{\ln z_{i}+\ln \left(1-z_{n+1-i}\right)\right\} \quad \text { for AD test. }
\end{aligned}
$$

It is worth mentioning that the collection of optimal thresholds for individual components may not be the optimal threshold combination for the mixture model. An additional procedure is needed to find an optimal combination of the individual thresholds. Again, a goodness-of-fit test is used to make the decision. However, only a non-parametric test is reasonable to be used due to the feature of mixture model: the Kolmogorov-Smirnov (KS) test has been chosen in this work. In statistics, the KS test is a non-parametric test and qualifies a distance between the empirical distribution function of the sample and the cumulative distribution function of the reference distribution. In addition, the generalized Pareto distribution has an important property that will be used to find the optimal combination of thresholds. If excesses of a sample over the optimal threshold, 
$u_{0}$, can be reasonably modelled by a GPD with shape parameter $\xi$ and $\sigma_{0}$, then the excesses over thresholds larger than the optimum will follow GPDs with same shape parameter $\xi$ but different scale parameter, $\sigma_{u}$ that linearly depends on the threshold value $\sigma_{u}=\sigma_{0}+\xi\left(u-u_{0}\right)$. Therefore, the solution is to find a set of $u_{1}, \cdots, u_{m}$ that satisfy:

$$
D_{n}=\sup \left\{F_{n}(x)-F\left(x ; u_{1}, \cdots, u_{m}\right)\right\} .
$$

\section{The estimation of GPD parameters}

Estimating the distribution parameters of GPD is another decisive point that influences the performance of the MPOT method. Various estimators have been proposed to estimate the parameters of GPD. The applicability of a certain method depends on the features of the considered data. A comprehensive review and qualitative comparison of different parameter estimation methods has been provided by (de Zea Bermudez and Kotz 2010), and a quantitative study has been conducted in (Zhou 2013) to evaluate the performance of various parameter estimation methods when applying peaks-over-threshold method on traffic load effect data. The method of moment (MM), the power weighted moment method (PWM) and the maximum likelihood method (ML) are commonly used in the literature. It has been widely accepted that the maximum distribution of bridge traffic load effects belongs to an upper bounded Weibull distribution which has a shape parameter $\xi<0$. Hence, the MM, PWM and ML methods are suitable to traffic load effects. In addition, the minimum density power divergence (MDPD) method is used in this work due to its excellent performance in the case of contaminated data (Juarez and Schucany 2004).

\section{TRAFFIC DATA AND BRIDGE TRAFFIC LOAD EFFECTS}




\section{Description of Weigh-in-Motion traffic data}

Traffic data from the A9 motorway near Saint-Jean-de-Védas (SJDV), in southeastern France, was used in this study. Weights and dimensions of trucks, which travelled in the slow and fast lanes in one direction of the 6-lane motorway, were recorded by using a piezo-ceramic Weigh-in-Motion (WIM) system from January 2010 to May 2010. A total number of 581,011 trucks representing traffic of 86 days were drawn from the original data by excluding unreasonable recordings, weekends and system inactivity days. The traffic composition displayed in Fig.1a shows that the 5-axle truck is the dominant type of truck on this site representing $76.4 \%$ in traffic volume. The histogram of gross vehicle weight (GVW) is presented in Fig.1b. To see the contribution from each type of truck, a stacked plot is given. It can be seen that the 5-axle truck governs the leading mode of the GVW histogram.

\section{Traffic loading Monte Carlo simulation}

If traffic data can be recorded by WIM for a sufficiently long period of time, such as a year, then the load effects induced by the measured traffic can be directly used to estimate the extreme load effect. Long term data, however, are not always available, due to the limitation of storage for huge amount of data for continuous recording, the problem of the equipment, the limitation of budget for conducting long term measuring, etc. Using limited data to predict extreme value distribution is thus a common situation in practice. The estimate of characteristic value may have large variance if extrapolation is based on limited data. Hybrid method that integrates extreme value analysis approaches with traffic simulation techniques is a practical solution. Using microscopic traffic simulation techniques to generate long-term traffic loads or load effects has been demonstrated as an efficient and accurate approach to study bridge traffic load effect in recent years 
(O'Connor and O'Brien 2005; Chen and Wu 2011; Enright and O'Brien 2012).

In the present study, a simulation program is developed to generate virtual traffic and to calculate traffic load effects on bridges. The basic principle is to generate traffic flow with the same features as those extracted from measured traffic data, such as aforementioned 86 days' WIM data. This is realized in following steps:

1. Calculating traffic composition: In this study, vehicles are categorized into classes according to their silhouettes as illustrated in Fig.2.

2. Establishing statistical models for characteristics of each class of vehicle, including gross vehicle weight (GVW), distribution of GVW to individual axle or axle group, vehicle speed, vehicle configuration in terms of axle spacing and vehicle length, and lateral position of the vehicle in the lane. The best fit is selected among the normal, bi- and tri-modal normal distribution.

3. Establishing vehicle moving model: Time headway distribution model, which describes the time distance between the rear axle of the front truck and the front axle of the following truck, is fundamental to traffic flow modelling in traffic simulation. A refined hourly truck flow rate depended headway model proposed by O'Brien and Caprani (2005) is adopted in the present study. Headways of less than 4 seconds are modelled using quadratic curves for different flow rates, and a negative exponential distribution is used for larger headways.

4. Simulating traffic flow: Assume the simulation program started at time $t$, a group of $n_{t}$ vehicles is generated by using headway model in step (3); each vehicle of these $n_{t}$ vehicles is randomly assigned a vehicle class with the traffic composition information that is a uniform distributed random 
variable ranging from 0 to 1 , and the vehicle characteristics are generated according to the assigned class.

5. Calculating load effects: Once the traffic data is generated, it is passed to the load effect calculation subroutine. The calculation is activated when a vehicle arrives on the bridge, then this vehicle is assumed as leading vehicle and passes the bridge in a time step $\Delta t$.

At each step, the program searches and counts the number of vehicles, $N$, on the bridge. The load effect, $L E\left(t_{n}\right)$, at time, $t_{n}$, induced by these $N$ vehicles can be obtained by using:

$$
L E\left(t_{n}\right)=\sum_{j=1}^{N} \sum_{k=1}^{n_{j}} \phi S_{i}\left(x_{j}^{k}, y_{j}^{k}\right) P_{j}^{k},
$$

where:

$N$ : number of vehicles on the bridge,

$n_{j}$ : number of axles of the $j$ th vehicle,

$\phi$ : dynamic amplification factor,

$S_{i}$ : influence surface for load effect of interest produced by a unit load of $i$-th type of tyre,

$x_{j}^{k}$ : longitudinal position of the $k$-th axle of the $j$-th vehicle

$$
x_{j}^{k}=v_{j} \cdot\left(t_{n}-t_{j}^{0}\right)-d_{j}^{k}
$$

$v_{j}$ : speed of $j$ th vehicle,

$t_{j}^{0}$ : arrival time of the first axle of the $j$ th vehicle, when passing over the position $x=0$, 
$d_{j}^{k}$ : distance between steering axle and the $k$ th axle of the $j$ th vehicle,

$y_{j}^{k}$ : transversal position of the $k$-th axle of the $j$-th vehicle,

$P_{j}^{k}$ : load of the $k$-th axle of the $j$ th vehicle.

\section{Classifying load effects by loading event}

Recording traffic load effects and loading events simultaneously, Fig.3 shows that several single truck loading events have induced a larger load effect than those induced by 2-truck loading events. In order to use all possible relatively large load effects efficiently, the full time history of effects induced by traffic passing over the bridge is retained first, then the local extremes and corresponding types of loading events (comprising the number of trucks) are identified. Fig. 4 illustrates such a process, the time history of the traffic load effect is drawn in blue line and the local extremes are marked with red stars:

1. The process starts with a single loading event when the first truck arrives the bridge.

2. Then another truck (2nd truck) arrives on the bridge generating a 2-truck loading event.

3. The first arrived truck leaves the bridge and the loading becomes a single truck event again.

4. Then a new truck (3rd truck) enters the bridge and the loading becomes a 2-truck event again,

5. The 2nd arrived truck exits the bridge (single loading event),

6. Then a new truck (4th truck) arrives so that a new 2-truck loading event is generated,

7. Finally the 3rd truck exits the bridge and the loading event is a single truck loading event again. 
In this process, a total of four trucks has arrived on the bridge and produced 4 extreme single truck loading events and 3 extreme two-truck loading events. The local extreme for each loading event is identified and marked in Fig.4. Using this procedure, local extremes for various types of loading event are identified. Fig.5 shows histograms of traffic load effects induced by simulated traffic for illustration purpose, and it can be seen that local extremes induced by different types of loading events are not identically distributed. The classical extreme value theory can thus not be directly applied to these mixed data as it requires data of independent and identical distribution.

Previous studies have demonstrated that three types of load effects are critical for short to median length bridges: (I1) bending moment at mid-span and (I2) shear force at end-support of a simply supported bridge, and (I3) hogging moment at middle support of a two-span continuous bridge. In this study, these three types of load effects are studied with span lengths of $20 \mathrm{~m}, 30 \mathrm{~m}, 40 \mathrm{~m}$ and 50 $\mathrm{m}$. Considering the time consumption, 1500-day's traffic data were generated by the developed traffic simulation program using statistical inputs extracted from SJDV traffic data. For the three types of load effects, six categories of loading events have been identified from the simulation. These six categories of truck arrangements are 1-truck, 2-truck, 3-truck, 4-truck, 5-truck, and 6-truck loading events. It should be noted that the 1-truck case includes situations from only one axle of the truck to the whole truck being on the bridge. Similarly, 2-truck loading events include all possible combinations of two trucks, from both trucks having only one axle on the bridge to both trucks having all axles on the bridge simultaneously. This is also the case for all loading types.

Two sets of loading event composition are listed in Table 1 for the three types of load effects, with four types of bridge lengths. The first group is for load effects over 90th percentile, and the second group is for load effects above 95th 
percentile. Fig. 6 shows that the governing type of loading event changes with increased bridge length. For a bridge length of $20 \mathrm{~m}, 2$-truck and 3-truck loading events govern the upper tail. For a bridge length of $30 \mathrm{~m}$, it can be seen from Fig. 6 that the governing event is 3-truck loading event. For bridge lengths of 40 and $50 \mathrm{~m}, 3$-truck events are still the governing but some 4- and 5-truck events occur at the upper end of the simulation period. In addition, the composition of loading events are different between the data over 90th percentile and those over 95th percentile. In general, it demonstrates the importance to classify the load effects by loading events in predicting extreme value distribution or characteristic value.

\section{EVALUATING THE PERFORMANCE OF THE MPOT APPROACH}

To show how the MPOT method works for realistic bridge traffic load effects, two numerical studies have been conducted and are reported in this section. The first example is to examine the performance of the MPOT method for a set of data generated from a mixture normal distribution, and the second example is to evaluate the MPOT method for bridge traffic load effects generated by Monte Carlo traffic microsimulation. In both examples, a comparison of the relative accuracy of the present MPOT and of the conventional peaks-over-threshold (CPOT) is performed.

\section{Theoretical example}

The normal distribution is widely used in bridge engineering: for example gross vehicle weights are usually modelled by normal distribution or mixture normal distribution. In the first example, the performance of MPOT method is evaluated by using a random event having a parent distribution of mixture normal distribution with two components, $F(X<x)=\varphi_{1} \Phi\left(\frac{x-\mu_{1}}{\sigma_{1}}\right)+\varphi_{2} \Phi\left(\frac{x-\mu_{2}}{\sigma_{2}}\right)$. The core distribution is $N(420,30)$ with the relative frequency of occurrence $\varphi_{1}=0.9$, 
and the "contaminating" distribution is $N(380,45)$ with the relative frequency of $\varphi_{2}=0.1$. Assuming a thousand events of this type occurring every day, three thousand days' events are simulated with a total of $n=(3000 \times 1000)=$ $3,000,000$-elements sample. In the simulation process, values from the $N(420,30)$ are denoted as event one, while those from the $N(380,45)$ are denoted as event two. These 3,000,000 sample are thus classified into two groups.

To approximate the upper tail of the distribution of the simulated sample, the two aforementioned CPOT and MPOT methods are applied. For the CPOT method, an optimal GPD is needed to be found, while for the MPOT method two optimal GPDs with one for each subgroup of events are required. The goodnessof-fit based threshold selection approach is used first to select the optimal threshold, then the GPD parameters for the exceedances are estimated by using the four previously mentioned estimators. Following this procedure, the threshold and GPD parameter estimates for the CPOT are obtained and tabulated in Table 2, and the corresponding results for the MPOT method are listed in Table 3.

Using these estimates, the upper tail distribution can be obtained from Eq.(3) for CPOT and Eq.(9) for MPOT. They are shown in a log-scale plot in Fig.7 along with the empirical distribution function of the sample. It can be seen that both CPOT and MPOT methods capture the main part of the distribution very well, but the discrepancy between empirical distribution and fitted distribution becomes larger when getting close to the upper tail. The CDF obtained from MPOT captures the upper tail with significantly less bias than that from the CPOT. Indeed, the MPOT follows the trend of the data, while the CPOT strongly deviates. By using the estimates of GPD, the quantile or characteristic values for a certain return period can be calculated from Eq.(4) for CPOT or Eq.(10) for MPOT. Fig. 8 compares the characteristic values for a return period of 100-year 
calculated with CPOT and MPOT methods with the real one (which is known because the underlying distribution is known). It indicates that both approaches have good performance on quantile estimation, with maximum error less than $2 \%$. The return levels estimated with conventional method are even much closer to the true value.

For reliability analysis, the maximum value distribution of load effects is required. After obtaining the upper tail distribution, it is straightforward to calculate the maximum value distribution function using $F^{n}(x)$. The CDFs of maximum value distribution with CPOT and MPOT methods are displayed in a Gumbel plot in Fig.9, where the true distribution is given as well. It can be seen that the MPOT based maximum value distribution matches the true distribution well, while the CPOT based maximum value distribution differs from the true distribution, particularly at the upper tail.

Although the CPOT can provide a relatively accurate estimate of characteristic value, especially for low return period, as the advanced MPOT method, it can not predict the upper tail of the distribution in sufficient accuracy as significant deviation is found in maximum value distribution when comparing with the bench mark. It is of particular importance to estimate the maximum value distribution for reliability-based structural assessment. It therefore illustrates the importance to consider the inherent distribution for load effects.

\section{Simulated traffic load effect example}

The previous simple example showed that the MPOT method has better performance than the CPOT method when the data are not identically distributed. Now we will evaluate its performance for bridge traffic load effects, which are generated by the previously mentioned microscopic Monte Carlo traffic simulation program. Table 1 has shown that the upper tail of distribution for bridge traffic 
load effects consists of contributions from different loading events, and Fig.5 has displayed that load effects from different loading events have various distribution features in terms of distribution type or parameters. To show how these features influence the distribution function estimation or high quantile prediction and to demonstrate the advantage of the proposed method, a comparative study between the CPOT method and the MPOT method is performed.

To exclude the influence of the threshold selection, we firstly conducted the comparison with fixed thresholds at 90th, 92nd, 94th, 96th, and 98th percentiles. Again, the estimators of MM, PWM, ML, and MDPD are used to estimate the distribution parameters for the involved GPDs. We used the graphical method to evaluate the performance of MPOT and CPOT methods. For instance, Fig. 10 shows the comparison between CPOT method and MPOT method for bending moment at the mid-span of a simply-supported bridge with span of $40 \mathrm{~m}$ I1 load effect, and the distribution parameters are estimated by ML method. The graphs on the left in Fig.10 illustrate the empirical survival function (black dots) fitted function with CPOT estimates (red solid lines) and with mixture POT estimates (green dash lines) for various thresholds, while the graphs on the right side show corresponding these results in a logarithm scale plot. It can be seen that the MPOT method approximates the excesses over threshold with good accuracy, while the CPOT method approximates the majority of the data well but has poor approximation for the high tail. It is commonly accepted that the high tail is extremely important in the extreme value analysis such as quantile estimation. A quantitative method has been adopted to compare the performance of the two methods. The results of root-mean-square-error reported in Table 4 confirm that the MPOT method improves the modelling as a majority of the values for MPOT are smaller than those for CPOT. Therefore, the MPOT has better performance than the CPOT method in capturing the upper tail of the distribution. 
This preliminary study has demonstrated that the MPOT method has the potential to provide more accurate prediction than the CPOT method. When studying bridge traffic load effects, the prediction of characteristic values for long return periods, such as the 1000-year characteristic value for traffic load model in Eurocode (CEN 2003), is a critical issue. Here we will illustrate the difference between CPOT and MPOT on this characteristic value prediction. Except for these two GPD based methods, the comparison also includes the GEV distribution based BM method. In the preliminary study, fixed thresholds are used to compare the performance of CPOT and MPOT methods under consistent conditions. But it should be noted that a fixed threshold may not be optimal to approximate the upper tail distribution. Thus, in the following study, threshold for each GPD is selected by using the goodness-of-fit statistics based automatic method for both CPOT and MPOT methods. With an illustration purpose, the selected optimal threshold and corresponding distribution parameters for each component of the mixture distribution are listed in Table 5 for the I1 load effect with bridge length of $40 \mathrm{~m}$. The tail distribution consists of load effects from 2-truck, 3-truck and 4-truck loading events, thus three sets of threshold and parameters have to be estimated. It can be seen from the results that each component has different tail distribution. For instance, distribution for load effects resulting from 2-truck loading events has a Pareto distribution with shape parameter $\xi>0$, while those from 3 -truck loading events and 4-truck loading events have type II Pareto distribution with $\xi<0$. Similar procedures are applied to other load effect cases, then the optimal threshold and corresponding distribution parameters are obtained. For the BM method, daily maxima are identified from the simulated load effects, then GEV distribution is fitted to each set of daily maxima.

For the load effects I1, I2 and I3 with span lengths of $20 \mathrm{~m}, 30 \mathrm{~m}, 40 \mathrm{~m}$ and $50 \mathrm{~m}$, 
the 100-year and 1000-year return period characteristic values are calculated by the BM, CPOT and MPOT approaches. Results from the BM and CPOT methods are given in Table 6 for characteristic values for 100-year return period and in Table 7 for characteristic values for 1000-year return period in terms of relative difference with respect to the corresponding results from the MPOT method. The differences between conventional and mixture estimates are smaller for 100-year return level than for 1000-year return level. For example, the difference between the convention method and the proposed method for 100-year return level of load effect I1 with span of $30 \mathrm{~m}$ shown in Table 6 is around $-6.31 \%$ for MM case, while the difference for 1000-year return level in Table 7 is around $13.5 \%$. It confirms the common impression that the extrapolation to remote future is not stable. As expected, the difference between conventional method and mixture method is smaller for load effects for shorter spans, either the BM or the POT. For instance, the difference is $-8.49 \%$ for BM for 100-year return level of load effect 11 at length of $20 \mathrm{~m}$ in Table 6 , but it increases to about $17 \%$ at span length of $50 \mathrm{~m}$. The composition of loading events becoming more complex when span length increases, and more types of loading events thus become the governing loading events. Among the three types of load effects, the performances of the methods are different. The differences are larger for load effects of I3 than for the other two. As stated in Harman and Davenport (1979), the load effect of I3 is more sensitive to the multiple presence of trucks. This shows that the differences for return level of type I3 load effect between conventional method and mixture method becomes larger with the increase of span length.

To further demonstrate the accuracy of the proposed MPOT method, a comparison study between the present MPOT method and the composite distribution statistic (CDS) approach proposed by (Caprani et al. 2008), which fit GEV distribution to block maxima for load effects resulting from same loading event, has 
been performed to predict the characteristic values for 100-year return period and 1000-year period. The relative differences between these two approaches are given in Table 8. The two methods seem to provide consistent results. In general, the differences are less than $10 \%$, it can be concluded that the two loading event depended methods have similar performance. However, it is also clear that some of the differences are significant, especially for longer span lengths.

It is clear from Fig.10 that the CPOT method is strongly governed by the relative frequency extremes, it thus results in the upper tail with less observed extremes poorly fitted. While the proposed MPOT method considering the contribution by type of loading event that results in a well captured tail. Quantitative comparison in terms of characteristic value for 100- and 1000-year return period further demonstrates the difference between the two methods. Due to the lack of sufficient long-term measured traffic data, although it is impossible to provide an directly comparison between predict method and measurement, the comparison between the MPOT method and the CDS method provides confidence that the MPOT method can provide sufficiently accurate prediction.

\section{CONCLUSIONS}

Special caution should be taken when estimating the high quantile or finding the extreme value distribution for bridge traffic load effects. A novel method is proposed in the present paper to study extreme value distribution of bridge traffic load effects and properly predict the characteristic values for long return periods. The proposed method is based on the generalized Pareto distribution as the classic Peaks-over-Threshold method. But conversely to the GPD which is seldom fitted to load effects resulting from the same loading event defined by number of simultaneously involved trucks/vehicles, since bridge traffic load effects generally result from different loading events, the proposed method accounts for various 
numbers of simultaneous trucks/vehicles on the bridges. Thus, the upper tail of the load effect distribution can be approximated by a mixture generalized distribution, and the method is thus named Mixture Peaks-over-Threshold Approach. Numerical studies have been conducted to demonstrate the capability of the proposed method in predicting characteristic values and extreme value distribution for bridge traffic load effects. In a theoretical example with known distribution, comparison between conventional extreme value estimation methods and the proposed method shows that the proposed MPOT method has better performance to capture the upper tail of the parent distribution and the maximum value distribution. In the traffic load effects example, the differences can be seen between the conventional methods and the proposed method for predicting characteristic values. Consistent results have been obtained from the proposed MPOT method and the composite statistic distribution method. It is believed that the proposed MPOT method provides more accurate and reasonable prediction as it considers the non-identically distributed nature of load effects.

\section{ACKNOWLEDGMENT}

The authors gratefully acknowledge the financial support provided for this study by the Marie Curie Initial Training Network TEAM (Training in European Asset Management) project that has been funded by the European Community's 7th Framework Programme.

\section{REFERENCES}

Bailey, S. F. and Bez, R. (1999). "Site specific probability distribution of extreme traffic action effects." Probabilistic Engineering Mechanics, 14(12), 19-26.

Balkema, A. A. and de Haan, L. (1974). "Residual life time at great age." The Annals of Probability, 792-804. 
Cai, C. S. and Chen, S. R. (2004). "Framework of vehicle--bridge-wind dynamic analysis." Journal of Wind Engineering and Industrial Aerodynamics, 92(78), 579-607.

Caprani, C. C., O’Brien, E. J., and McLachlan, G. J. (2008). "Characteristic traffic load effects from a mixture of loading events on short to medium span bridges." Structural Safety, 30(5), 394-404.

CEN (2003). Eurocode 1: Actions on Structures - Part 2: Traffic Loads on Bridges. European Committee for Standardization, Brussels, Belgium.

Chen, S. R. and Wu, J. (2011). "Modeling stochastic live load for long-span bridge based on microscopic traffic flow simulation." Computers \& Structures, 89(910), 813-824.

Choulakian, V. and Stephens, M. A. (2001). "Goodness-of-fit tests for the generalized pareto distribution." Technometrics, 43(4), 478-484.

Coles, S. (2001). An introduction to statistical modeling of extreme values. Springer Series in Statistics. Springer-Verlag London Limited, London.

Cooper, D. I. (1997). "Development of short span bridge-specific assessment live loading." The safety of bridges, P. C. Das, ed. Thomas Telford, 64-89.

COST 345 (2002). Procedures required for assessing highway structures: final report. European Commission Directorale General Transport and Energy.

Cremona, C. (2001). "Optimal extrapolation of traffic load effects." Structural Safety, 23(1), 31-46.

de Haan, L. and Ferreira, A. (2006). Extreme Value Theory: An Introduction. Springer series in operations research and financial engineering. Springer.

de Zea Bermudez, P. and Kotz, S. (2010). "Parameter estimation of the generalized Pareto distribution - Part I \& II." Journal of Statistical Planning and Inference, 140, 1353-1388.

Desrosiers, R. and Grillo, R. (1973). Estimating the Frequency of Multiple Truck 
Loadings on Bridges: Final Report. University of Connecticut.

Enright, B., Carey, C., and Caprani, C. (2013). "Microsimulation evaluation of Eurocode load model for American long-span bridges." Journal of Bridge Engineering, 18(12), 1252-1260.

Enright, B. and O'Brien, E. J. (2012). "Monte Carlo simulation of extreme traffic loading on short and medium span bridges." Structure and Infrastructure Engineering, 1-16.

Frangopol, D., Strauss, A., and Kim, S. (2008). "Bridge reliability assessment based on monitoring." Journal of Bridge Engineering, 13(3), 258-270.

Fu, G., Liu, L., and Bowman, M. (2011). "Multiple presence factor for truck load on highway bridges." Journal of Bridge Engineering, 18(3), 240-249.

Fu, G. and You, J. (2009). "Truck loads and bridge capacity evaluation in China." Journal of Bridge Engineering, 14(5), 327-335.

Ghosh, J., Caprani, C., and Padgett, J. (2013). "Influence of traffic loading on the seismic reliability assessment of highway bridge structures." Journal of Bridge Engineering, 19(3), 04013009.

Ghosn, M. and Moses, F. (2000). "Effect of changing truck weight regulations on U.S. bridge network." Journal of Bridge Engineering, 5(4), 304-310.

Gindy, M. and Nassif, H. H. (2007). "Multiple presence statistics for bridge live load based on weigh-in-motion data." Transportation Research Record: Journal of the Transportation Research Board, 125-135.

Harman, D. J. and Davenport, A. G. (1979). "A statistical approach to traffic loading on highway bridges." Canadian Journal of Civil Engineering, 6(4), 494-513.

Juarez, S. F. and Schucany, W. R. (2004). "Robust and efficient estimation for the generalized pareto distribution." EXTREMES, 7, 237-251.

Leadbetter, R. M., Lindgren, G., and Rootzen, H. (1983). Extremes and related 
properties of random sequences and processes. Springer-Verlag, New York Heidelberg Berlin.

Li, S., Zhu, S., Xu, Y.-L., Chen, Z.-W., and Li, H. (2012). "Long-term condition assessment of suspenders under traffic loads based on structural monitoring system: Application to the Tsing Ma Bridge." Structural Control and Health Monitoring, 19(1), 82-101.

Madsen, H., Rasmussen, P. F., and Rosbjerg, D. (1997). "Comparison of annual maximum series and partial duration series methods for modeling extreme hydrologic events: 1. at-site modeling." Water Resources Research, 33(4), 747757.

Messervey, T. B., Frangopol, D. M., and Casciati, S. (2010). "Application of the statistics of extremes to the reliability assessment and performance prediction of monitored highway bridges." Structure and Infrastructure Engineering, 7(12), 87-99.

Nowak, A. S. (1993). "Live load model for highway bridges." Structural Safety, $13,53-66$.

O'Brien, E. and Caprani, C. C. (2005). "Headway modelling for traffic load assessment of short to medium span bridges." The Structural Engineer, 86(16), $33-36$.

O’Brien, E., Schmidt, F., Hajializadeh, D., Zhou, X. Y., Enright, B., Caprani, C. C., Wilson, S., and Sheils, E. (2015). "A review of probabilistic methods of assessment of load effects in bridges." Structural Safety, 53, 44-56.

O'Brien, E. J., Bordallo-Ruiz, A., and Enright, B. (2014). "Lifetime maximum load effects on short-span bridges subject to growing traffic volumes." Structural Safety, 50, 113-122.

O’Brien, E. J., Sloan, D. T., Bulter, K. M., and Kirkpatrick, J. (1995). "Traffic load 'fingerprinting' of bridges for assessment purposes." The Structural Engi- 
neer, 73(19), 320-324.

O'Connor, A. and O'Brien, E. J. (2005). "Traffic load modelling and factors influencing the accuracy of predicted extremes." Canadian Journal of Civil Engineering, 32(1), 270-278.

Park, H. W. and Sohn, H. (2006). "Parameter estimation of the generalized extreme value distribution for structural health monitoring." Probabilistic Engineering Mechanics, 21(4), 366-376.

Pickands III, J. (1975). "Statistical inference using extreme order statistics." The Annals of Statistics, 3(1), 119-131.

Righiniotis, T. D. (2006). "Effects of increasing traffic loads on the fatigue reliability of a typical welded bridge detail." International Journal of Fatigue, 28(8), 873-880.

Scarrott, C. and MacDonald, A. (2012). "A review of extreme value threshold estimation and uncertainty quantification." REVSTAT - Statistical Journal, 10(1), 33-60.

Sivakumar, B., Ghosn, M., Moses, F., and TranSystems Corporation (2011). "Protocols for collecting and using traffic data in bridge design." National Cooperative Highway Research Program (NCHRP) Report 683, Lichtenstein Consulting Engineers, Inc., Washington, D. C.

Tong, G., Aiqun, L., and Jianhui, L. (2008). "Fatigue life prediction of welded joints in orthotropic steel decks considering temperature effect and increasing traffic flow." Structural Health Monitoring, 7(3), 189-202.

Zhou, X., Schmidt, F., Toutlemonde, F., and Jacob, B. (2014). "Applying Weighin-Motion traffic data to reliability based assessment of bridge structures." Safety, Reliability, Risk and Life-Cycle Performance of Structures and Infrastructures. CRC Press, 3831-3838.

Zhou, X.-Y. (2013). "Statistical analysis of traffic loads and their effects on 
${ }_{657}$ bridges using Weigh-in-Motion data collected in France," Ph.D. thesis, Uni658 versité Paris-Est.

${ }_{659}$ Zhu, B. and Frangopol, D. (2012). "Risk-based approach for optimum mainte${ }_{660}$ nance of bridges under traffic and earthquake loads." Journal of Structural $661 \quad$ Engineering, 139(3), 422-434. 


\section{List of Tables}

1 Probabilities for six categories of loading events for data above $90^{\text {th }}$ and $95^{t h}$ percentile ...................... 30

$2 \quad$ Parameter estimates for the CPOT method by various estimators 31

$3 \quad$ Parameter estimates for the MPOT method by various estimators 31

4 Root mean square error at various thresholds . . . . . . . . . . . . 31

5 Optimal threshold selection for I1 (bending moment at mid-span of simply supported bridge) with bridge length of 40m . . . . . 32

6 Percentage difference of 100-year return level between conventional and mixture method $(\%)$. . . . . . . . . . . . . . . 33

7 Difference in 1000-year return level between conventional and mix-

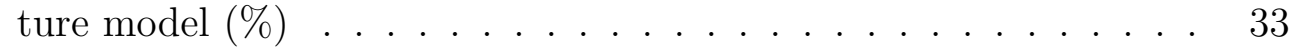

8 Difference (mixture POT vs. mixture GEV) . . . . . . . . . . . . 34 


\begin{tabular}{|c|c|c|c|c|c|c|c|c|c|c|c|c|c|}
\hline \multirow{3}{*}{$\begin{array}{l}\text { व } \\
0 \\
0\end{array}$} & $\stackrel{\oplus}{\oplus}$ & 1 & $\stackrel{0}{\ddot{0}}$ & $\left|\begin{array}{c}\hat{0} \\
\hat{N} \\
\infty \\
\infty\end{array}\right|$ & 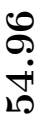 & חִ & 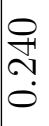 & & : & 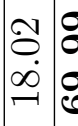 & 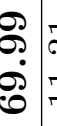 & 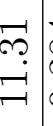 & 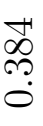 \\
\hline & 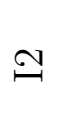 & $\begin{array}{r}\mathscr{P} \\
\stackrel{+}{+} \\
\stackrel{0}{0}\end{array}$ & $\begin{array}{l}\curvearrowright \\
\sim \\
\sim \\
\sim\end{array}$ & $\mid \begin{array}{l}\infty \\
0 \\
0 \\
0 \\
0\end{array}$ & $\overrightarrow{\dot{\rho}}$ & $\underset{\sim}{\stackrel{\sim}{+}}$ & 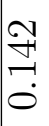 & $\begin{array}{l}20 \\
\infty \\
0 \\
0 \\
0\end{array}$ & 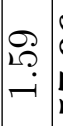 & 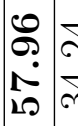 & 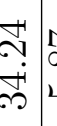 & & 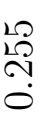 \\
\hline & $\Xi$ & \begin{tabular}{l}
10 \\
120 \\
\hdashline-1 \\
0 \\
0
\end{tabular} & $\begin{array}{l}\stackrel{\curvearrowright}{\sim} \\
\sim\end{array}$ & $\begin{array}{l}\boldsymbol{N} \\
\mathfrak{N} \\
\mathfrak{N}\end{array}$ & $\stackrel{m}{\stackrel{\leftrightarrow}{\sim}}$ & $\stackrel{\sim}{\sim}$ & 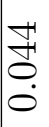 & 1 & $\mid$ & 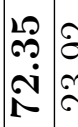 & 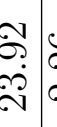 & 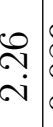 & $\begin{array}{l}\mathscr{O} \\
\infty \\
0 \\
0 \\
0\end{array}$ \\
\hline \multirow{3}{*}{$\begin{array}{c}\Xi \\
O \\
O \\
+\end{array}$} & $\stackrel{\oplus}{\oplus}$ & 1 & 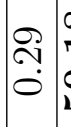 & $\mid$\begin{tabular}{c}
$\infty$ \\
\hdashline-1 \\
0 \\
10 \\
10
\end{tabular} & 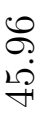 & $\begin{array}{l}1 \\
20 \\
\infty\end{array}$ & 1 & 1 & $\mid$ & 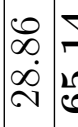 & $\begin{array}{l}7 \\
\\
\\
0\end{array}$ & ڤִ & I \\
\hline & 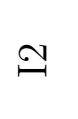 & $\begin{array}{l}20 \\
0 \\
0 \\
0 \\
0\end{array}$ & 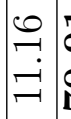 & $\mid \begin{array}{l}\sigma \\
\dot{\sigma} \\
\dot{\sigma}\end{array}$ & 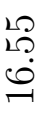 & חִ & 1 & $\begin{array}{l}\vec{\infty} \\
0 \\
0 \\
0\end{array}$ & 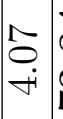 & 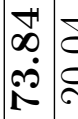 & $\begin{array}{ll}\vec{D} \\
\dot{\sigma} \\
\stackrel{\sigma}{\sigma}\end{array}$ & 尚 & 1 \\
\hline & $\Xi$ & $\begin{array}{l}\stackrel{N}{\vec{H}} \\
\stackrel{0}{0} \\
\stackrel{0}{*}\end{array}$ & $\begin{array}{l}\Re \\
\beth \\
\beth\end{array}$ & $\mid \begin{array}{c}0 \\
\varrho \\
\dot{1} \\
\end{array}$ & $\stackrel{P}{\stackrel{P}{\infty}}$ & $\stackrel{n}{\infty}$ & ' & $\begin{array}{l}\vec{J} \\
\dot{D} \\
\dot{0}\end{array}$ & $\mid \begin{array}{l}\stackrel{1}{S} \\
\sim \\
+ \\
+\end{array}$ & $\begin{array}{ll}\vec{J}^{\prime} & \alpha \\
\dot{J}_{\infty}^{+} & =\end{array}$ & $=$ & $\underset{+}{\stackrel{H}{*}}$ & 1 \\
\hline \multirow{3}{*}{ 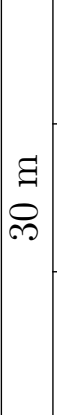 } & $\stackrel{\oplus}{\oplus}$ & $\begin{array}{l}0 \\
130 \\
0 \\
0 \\
0\end{array}$ & 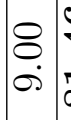 & $\begin{array}{c}0 \\
\overrightarrow{+} \\
\dot{\infty} \\
\infty\end{array}$ & ঙ্: & \begin{tabular}{l}
$\infty$ \\
$\stackrel{\infty}{+}$ \\
\hdashline \\
0
\end{tabular} & ' & $\begin{array}{l}m \\
\ddot{Z} \\
\dot{0}\end{array}$ & 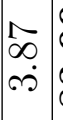 & $\begin{array}{ll}\mathscr{D} & \\
\infty & 1 \\
\infty & \end{array}$ & 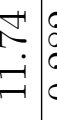 & $\begin{array}{l}\stackrel{N}{\infty} \\
\text { ָे } \\
0 \\
0\end{array}$ & 1 \\
\hline & $\stackrel{\sim}{\vartheta}$ & $\begin{array}{l}\hat{0} \\
0 \\
0 \\
0\end{array}$ & $\begin{array}{l}\overrightarrow{1} \\
\dot{2} \\
\ddot{\theta}\end{array}$ & 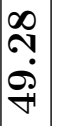 & $\vec{\sigma}$ & 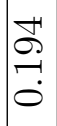 & ' & $\begin{array}{l}\Re \\
⿱ \\
0 \\
0 \\
0\end{array}$ & 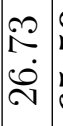 & \begin{tabular}{l|l}
10 \\
10 \\
10 \\
10 \\
0
\end{tabular} & $\stackrel{n}{r}$ & 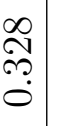 & ' \\
\hline & $\Xi$ & $\begin{array}{l}0 \\
1 \\
0 \\
0 \\
0\end{array}$ & $\begin{array}{l}10 \\
0 \\
0 \\
\infty \\
\infty\end{array}$ & $\begin{array}{l}1 \\
\infty \\
\dot{\infty} \\
\dot{1}\end{array}$ & $\begin{array}{c}0 \\
\underset{1}{1} \\
\dot{\infty}\end{array}$ & $\begin{array}{l}1 \\
\infty \\
0 \\
0 \\
0\end{array}$ & I & $\begin{array}{c}8 \\
0 \\
\dot{0} \\
0\end{array}$ & $\mid$ & 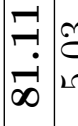 & 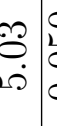 & \begin{tabular}{l}
0 \\
$\stackrel{10}{0}$ \\
\hdashline \\
0
\end{tabular} & I \\
\hline \multirow{3}{*}{$\begin{array}{l}\Xi \\
\stackrel{\sim}{\sim}\end{array}$} & $\stackrel{\oplus}{\sim}$ & $\begin{array}{l}\infty \\
0 \\
0 \\
0 \\
0 \\
0\end{array}$ & $\begin{array}{c}1 \\
\dot{D} \\
\dot{0}\end{array}$ & $\left|\begin{array}{c}0 \\
\infty \\
0 \\
\infty \\
\infty\end{array}\right|$ & 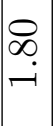 & I & ' & $\begin{array}{l}0 \\
\infty \\
-1 \\
0\end{array}$ & 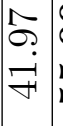 & $\begin{array}{c}8 \\
0 \\
010 \\
10\end{array}$ & $\begin{array}{l}\infty \\
\infty \\
\dot{\sigma}\end{array}$ & 1 & 1 \\
\hline & $\bumpeq$ & \begin{tabular}{c}
0 \\
\multirow{2}{0}{} \\
0 \\
0 \\
0
\end{tabular} & $\begin{array}{l}2 \\
+ \\
\dot{10} \\
\dot{10}\end{array}$ & $\left|\begin{array}{c}0 \\
N \\
\infty \\
\infty\end{array}\right|$ & $\stackrel{1}{10}$ & ' & ' & $\begin{array}{l}0 \\
N \\
0 \\
0\end{array}$ & $\left.\begin{array}{|l}\infty \\
\infty \\
\sim \\
\sim \\
\mathcal{F}\end{array}\right]$ & 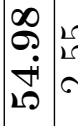 & חֶ & 1 & 1 \\
\hline & $\Xi$ & $\begin{array}{l}\stackrel{N}{\vec{O}} \\
\stackrel{0}{0}\end{array}$ & 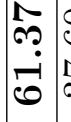 & $\begin{array}{l}\mathscr{\bullet} \\
\stackrel{0}{\infty} \\
\infty\end{array}$ & ஓ. & I & ' & $\begin{array}{l}\vec{H} \\
\mathscr{D} \\
0\end{array}$ & $\mid \begin{array}{c}\infty \\
\infty \\
\infty \\
\infty \\
\infty \\
\infty\end{array}$ & \begin{tabular}{l|l}
$\infty$ & 1 \\
$\infty$ & 1 \\
$\infty$ & \multicolumn{1}{c}{} \\
10 & -
\end{tabular} & 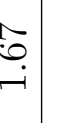 & 1 & 1 \\
\hline 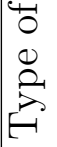 & 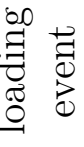 & 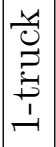 & 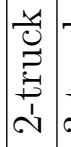 & 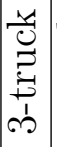 & 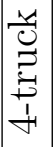 & فํ. & & 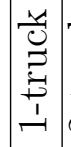 & 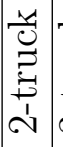 & 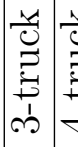 & 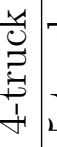 & 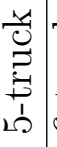 & 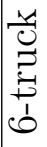 \\
\hline & & & & & & & & & & 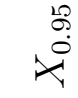 & & & \\
\hline
\end{tabular}


TABLE 2: Parameter estimates for the CPOT method by various estimators

\begin{tabular}{|c|c|c|c|c|c|}
\hline Estimator & Shape & Scale & Location & No. exceedances & KS, p-value \\
\hline MM & -0.0767 & 10.21 & 510.52 & 1321 & 0.8823 \\
\hline PWM & -0.0930 & 10.37 & 510.52 & 1321 & 0.9735 \\
\hline ML & -0.0583 & 10.03 & 510.52 & 1321 & 0.6936 \\
\hline MDPD & -0.0760 & 10.20 & 510.52 & 1321 & 0.8726 \\
\hline
\end{tabular}

TABLE 3: Parameter estimates for the MPOT method by various estimators

\begin{tabular}{|c|l|c|c|c|c|}
\hline \multirow{2}{*}{ Item } & \multirow{2}{*}{ Parameter } & \multicolumn{4}{|c|}{ Estimator } \\
\cline { 2 - 6 } & & MM & PWM & ML & MDPD \\
\hline \multirow{4}{*}{ Comp. 1 } & Shape, $\xi$ & -0.173 & -0.105 & -0.177 & -0.177 \\
\cline { 2 - 6 } & Scale, $\sigma$ & 9.9 & 10.0 & 10.0 & 10.0 \\
\cline { 2 - 6 } & Location, $\mu$ & 515.2 & 508.0 & 515.2 & 515.2 \\
\cline { 2 - 6 } & No. exceed. & 707 & 1500 & 707 & 707 \\
\cline { 2 - 6 } & KS p-value & 0.908 & 0.909 & 0.922 & 0.922 \\
\hline \multirow{4}{*}{ Comp. 2 2} & Shape, $\xi$ & -0.056 & -0.058 & -0.053 & -0.057 \\
\cline { 2 - 6 } & Scale, $\sigma$ & 15.9 & 16.0 & 15.9 & 16.0 \\
\cline { 2 - 6 } & Location, $\mu$ & 479.1 & 479.1 & 479.1 & 479.1 \\
\cline { 2 - 6 } & No. exceed. & 1371 & 1371 & 1371 & 1371 \\
\cline { 2 - 6 } & KS p-value & 0.926 & 0.903 & 0.945 & 0.918 \\
\hline Mixture & KS p-value & 0.964 & 0.866 & 0.979 & 0.974 \\
\hline
\end{tabular}

TABLE 4: Root mean square error at various thresholds

\begin{tabular}{|c|c|c|c|c|c|c|}
\hline Threshold & No. & Method & MM & PWM & ML & MDPD \\
\hline \multirow{2}{*}{$X_{0.90}$} & \multirow{2}{*}{6403} & CPOT & 0.0091 & 0.0083 & 0.0035 & 0.0066 \\
\cline { 3 - 7 } & & MPOT & $\mathbf{0 . 0 0 4}$ & $\mathbf{0 . 0 0 5 9}$ & $\mathbf{0 . 0 0 3 2}$ & $\mathbf{0 . 0 0 6 2}$ \\
\hline \multirow{2}{*}{$X_{0.92} 5122$} & CPOT & 0.0079 & 0.0079 & 0.0034 & 0.0063 \\
\cline { 3 - 7 } & & MPOT & $\mathbf{0 . 0 0 3 3}$ & $\mathbf{0 . 0 0 5 4}$ & $\mathbf{0 . 0 0 3 2}$ & 0.0065 \\
\hline \multirow{2}{*}{$X_{0.94} 3842$} & CPOT & 0.0099 & 0.0083 & 0.0064 & 0.0099 \\
\cline { 3 - 7 } & & MPOT & $\mathbf{0 . 0 0 6 1}$ & $\mathbf{0 . 0 0 7 9}$ & $\mathbf{0 . 0 0 4 2}$ & $\mathbf{0 . 0 0 7 1}$ \\
\hline \multirow{2}{*}{$X_{0.96} 2561$} & CPOT & 0.0095 & 0.0084 & 0.0048 & 0.0083 \\
\cline { 3 - 7 } & & MPOT & $\mathbf{0 . 0 0 5 1}$ & $\mathbf{0 . 0 0 7 1}$ & $\mathbf{0 . 0 0 3 9}$ & $\mathbf{0 . 0 0 6 9}$ \\
\hline \multirow{2}{*}{$X_{0.98}$} & \multirow{2}{*}{1281} & CPOT & 0.0086 & 0.0086 & 0.0041 & 0.0061 \\
\cline { 3 - 7 } & & MPOT & $\mathbf{0 . 0 0 3 5}$ & $\mathbf{0 . 0 0 5 9}$ & $\mathbf{0 . 0 0 3 3}$ & 0.0068 \\
\hline
\end{tabular}




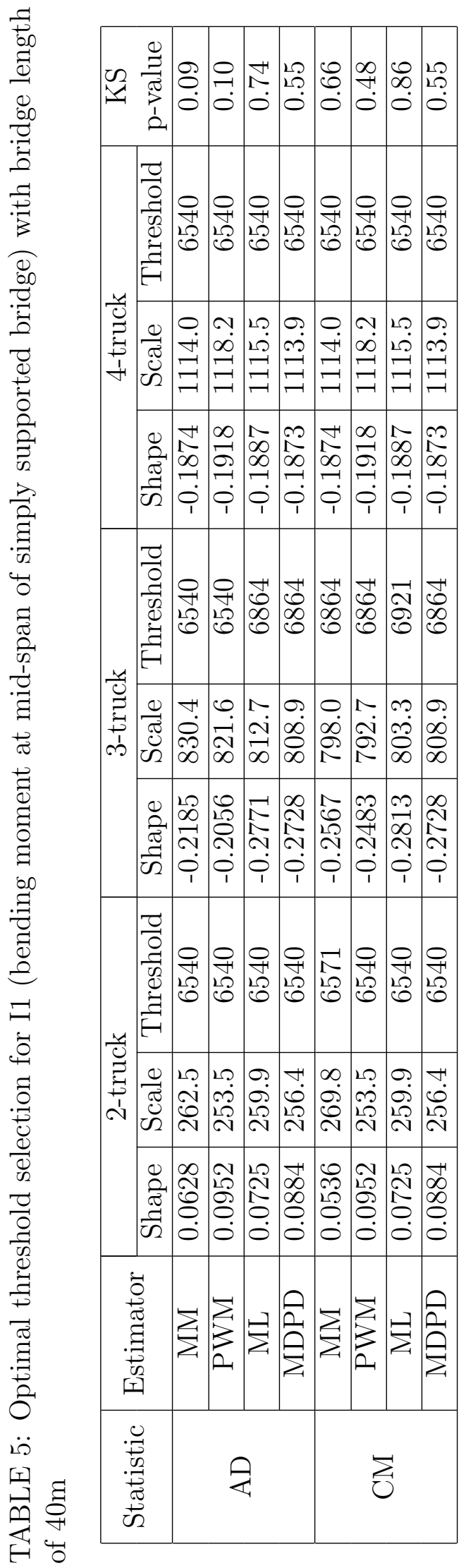


TABLE 6: Percentage difference of 100-year return level between conventional and mixture method (\%)

\begin{tabular}{|c|c|c|c|c|c|c|}
\hline Load & \multirow{2}{*}{$\begin{array}{c}\text { Leffect } \\
\text { Length }\end{array}$} & \multirow{2}{*}{ BM/GEV } & \multicolumn{4}{|c|}{ POT/GPD } \\
\cline { 3 - 7 } I1 & 20 & -8.49 & 0.11 & 0.43 & 0.19 & 0.17 \\
\cline { 2 - 7 } & 30 & -9.56 & -6.31 & -10.40 & -8.18 & -9.66 \\
\cline { 2 - 7 } & 40 & -14.63 & -8.27 & -7.90 & -1.82 & -7.19 \\
\cline { 2 - 7 } & 50 & -16.98 & 15.78 & -2.71 & 20.32 & 21.18 \\
\hline \multirow{4}{*}{ I2 } & 20 & 5.12 & -0.47 & 1.54 & 0.20 & 0.36 \\
\cline { 2 - 7 } & 30 & -20.60 & -3.02 & -0.32 & -6.33 & -3.66 \\
\cline { 2 - 7 } & 40 & -9.51 & -3.02 & -16.38 & -16.02 & -21.40 \\
\cline { 2 - 7 } & 50 & -11.22 & 0.08 & -2.73 & 1.20 & 1.04 \\
\hline \multirow{4}{*}{ I3 } & 20 & -29.92 & -4.55 & -7.11 & -1.63 & -4.30 \\
\cline { 2 - 7 } & 30 & -15.22 & -5.89 & -9.69 & -4.11 & -5.92 \\
\cline { 2 - 7 } & 40 & -8.28 & 5.76 & 20.09 & 16.59 & 23.89 \\
\cline { 2 - 7 } & 50 & -17.85 & 8.44 & 24.03 & 9.14 & 14.40 \\
\hline
\end{tabular}

TABLE 7: Difference in 1000-year return level between conventional and mixture model (\%)

\begin{tabular}{|c|c|c|c|c|c|c|}
\hline $\begin{array}{c}\text { Load } \\
\text { effect }\end{array}$ & \multirow{2}{*}{ Length } & \multirow{2}{*}{ BM/GEV } & \multicolumn{4}{|c|}{ POT/GPD } \\
\cline { 3 - 7 } & & & MM & PWM & ML & MDPD \\
\hline \multirow{4}{*}{ I1 } & 20 & -10.62 & 0.24 & 0.64 & 0.30 & 0.28 \\
\cline { 2 - 7 } & 30 & -16.20 & -13.50 & -22.30 & -22.38 & -24.88 \\
\cline { 2 - 7 } & 40 & -29.67 & -9.78 & -11.11 & -1.00 & -9.44 \\
\cline { 2 - 7 } & 50 & -36.45 & 34.53 & -1.39 & 44.65 & 46.16 \\
\hline \multirow{4}{*}{ I2 } & 20 & 8.65 & -0.80 & 1.93 & 0.05 & 0.28 \\
\cline { 2 - 7 } & 30 & -25.62 & -8.36 & -8.90 & -11.17 & -8.81 \\
\cline { 2 - 7 } & 40 & -11.39 & -4.48 & -36.71 & -36.18 & -42.90 \\
\cline { 2 - 7 } & 50 & -13.91 & 1.26 & -2.68 & 2.69 & 2.58 \\
\hline \multirow{4}{*}{ I3 } & 20 & -41.27 & -8.28 & -12.52 & -3.83 & -7.92 \\
\cline { 2 - 7 } & 30 & -17.82 & -7.10 & -13.42 & -6.72 & -10.99 \\
\cline { 2 - 7 } & 40 & -10.21 & 9.40 & 34.22 & 28.00 & 40.81 \\
\cline { 2 - 7 } & 50 & -17.65 & 15.34 & 40.60 & 16.50 & 24.94 \\
\hline
\end{tabular}


TABLE 8: Difference (mixture POT vs. mixture GEV)

\begin{tabular}{|c|c|c|c|c|c|c|c|c|c|}
\hline \multirow{2}{*}{$\begin{array}{l}\text { Load } \\
\text { effet }\end{array}$} & \multirow{2}{*}{ Length } & \multicolumn{4}{|c|}{ 100-year } & \multicolumn{4}{|c|}{ 1000-year } \\
\hline & & MM & PWM & ML & MDPD & MM & PWM & ML & MDPD \\
\hline \multirow{4}{*}{ I1 } & 20 & 0.43 & -0.65 & -0.57 & 0.89 & 0.48 & $\begin{array}{l}-0.74 \\
\end{array}$ & -0.65 & 1.02 \\
\hline & 30 & 2.14 & 0.13 & 1.22 & 9.21 & 8.13 & 8.84 & 11.73 & 17.17 \\
\hline & 40 & -0.14 & -0.05 & 0.25 & 0.46 & 1.80 & 0.14 & 1.32 & 8.82 \\
\hline & 50 & -1.78 & 0.50 & -0.10 & -1.38 & -2.44 & 0.70 & -0.14 & -1.85 \\
\hline \multirow{4}{*}{$\mathrm{I} 2$} & 20 & 0.13 & -1.91 & -1.38 & 1.74 & 0.08 & -2.45 & -1.79 & 2.14 \\
\hline & 30 & -3.81 & 3.98 & 0.60 & -3.03 & -0.92 & 3.74 & 0.41 & -3.95 \\
\hline & 40 & 16.27 & 15.95 & 23.47 & 32.22 & 51.41 & 50.41 & 67.44 & 87.83 \\
\hline & 50 & 2.79 & -0.85 & -0.99 & -1.23 & 3.93 & -1.07 & -1.35 & -1.53 \\
\hline \multirow{4}{*}{ I3 } & 20 & 5.27 & -4.12 & -0.21 & 15.40 & 8.39 & -6.18 & -0.32 & 25.15 \\
\hline & 30 & 3.34 & -1.77 & -0.06 & 2.40 & 6.06 & -0.29 & 4.23 & 13.06 \\
\hline & 40 & -0.07 & -0.72 & 0.00 & 5.57 & -0.12 & -1.28 & -0.03 & 10.09 \\
\hline & 50 & -5.15 & 1.49 & 0.36 & -2.09 & -7.19 & 2.18 & 0.51 & -2.80 \\
\hline
\end{tabular}




\section{List of Figures}

1 Characteristics of measured traffic data . . . . . . . . . . . 36

2 Classification of vehicles/trucks ............. 36

3 Time history of load effects . . . . . . . . . . . . . . . 37

4 Time history and local extreme . . . . . . . . . . . . . . . 37

$5 \quad$ Histogram of load effects due to various types of loading events . . 38

$6 \quad$ Probabilities for six types of loading events (left) over $90^{\text {th }}$ percentile 39

$7 \quad$ Gumbel scaled cumulative distribution probability plot. . . . . . . 39

8 Comparison of estimates of the characteristic values obtained from between CPOT and MPOT. . . . . . . . . . . . . . . 40

9 Extreme value distribution from CPOT and MPOT with true distribution. ....................... . . 40

10 Diagnosis plot for threshold excess model fitted to load effect. . . 41 


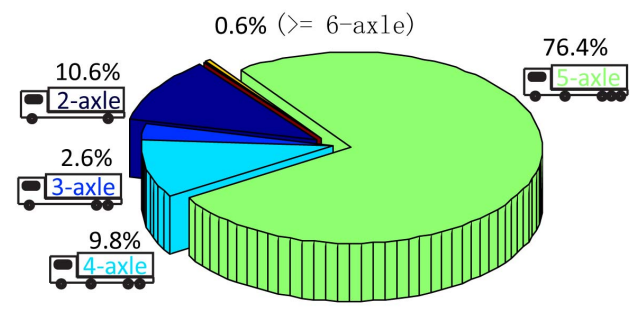

(a) Traffic composition

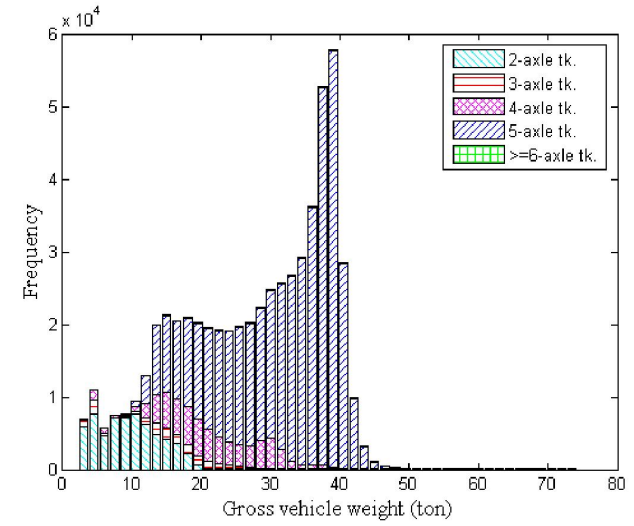

(b) Histogram of gross vehicle weight

FIG. 1: Characteristics of measured traffic data

\begin{tabular}{|c|c|c|c|c|}
\hline 2-axle & 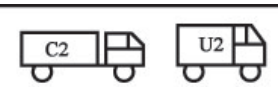 & & & \\
\hline 3-axle & \begin{tabular}{l|l} 
C38 \\
$\mathrm{Cg}$
\end{tabular} & م2281 & & 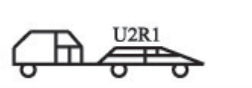 \\
\hline 4-axle & 5 C2R28 & 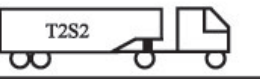 & & \\
\hline 5-axle & 500 c2838 & 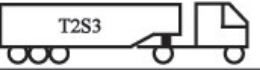 & [3BR2A] & [38в28 \\
\hline 6-axle & & 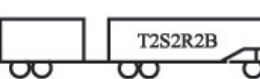 & & \\
\hline 7-axle & & \begin{tabular}{|c|c|c|} 
T352R2B \\
\end{tabular} & & \\
\hline 8-axle & & 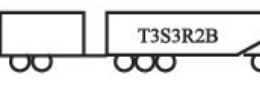 & & \\
\hline
\end{tabular}

FIG. 2: Classification of vehicles/trucks 


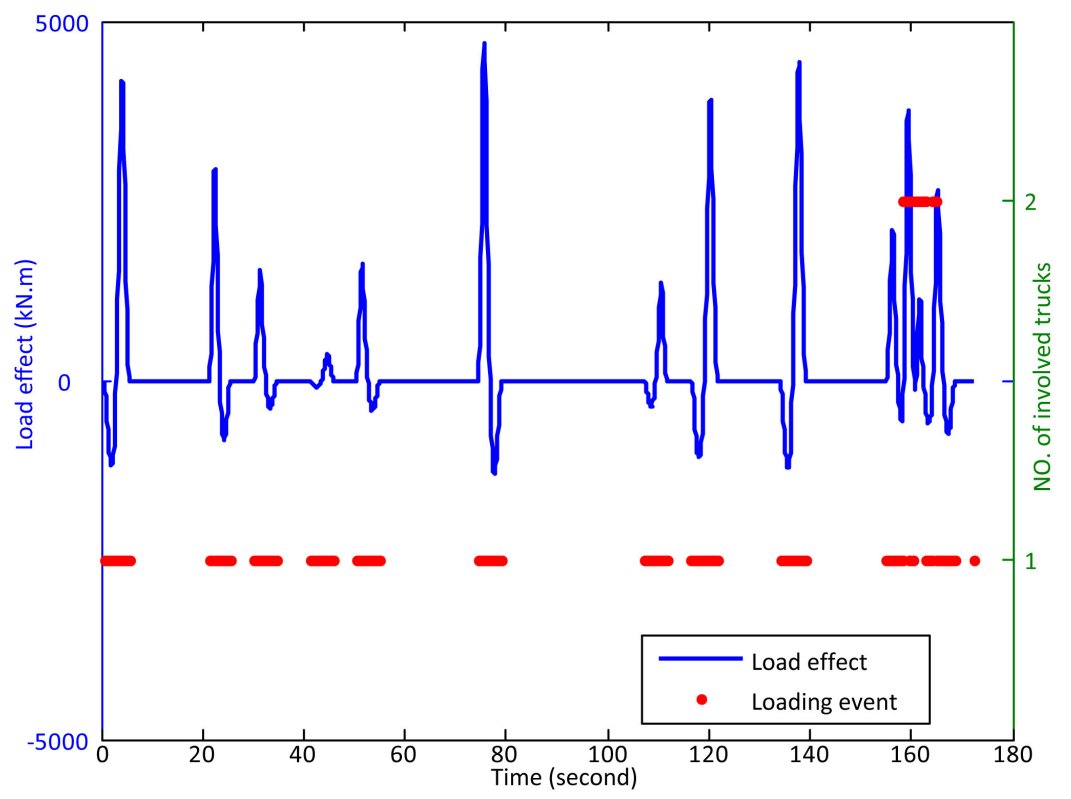

FIG. 3: Time history of load effects

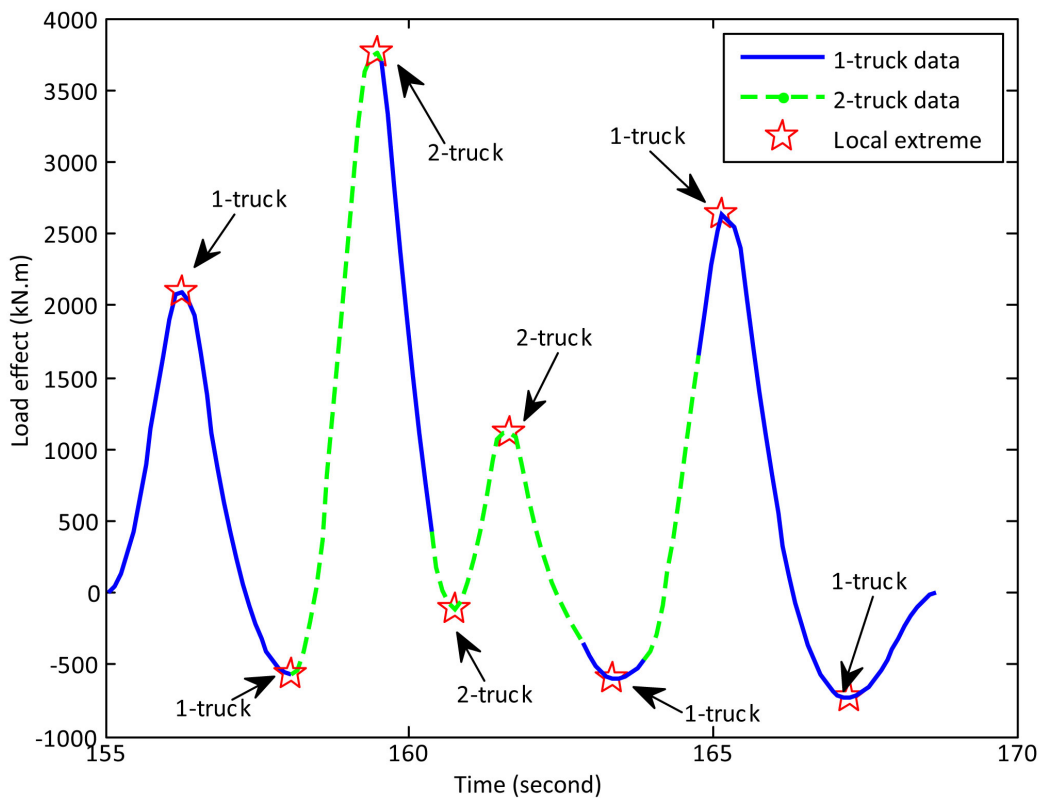

FIG. 4: Time history and local extreme 

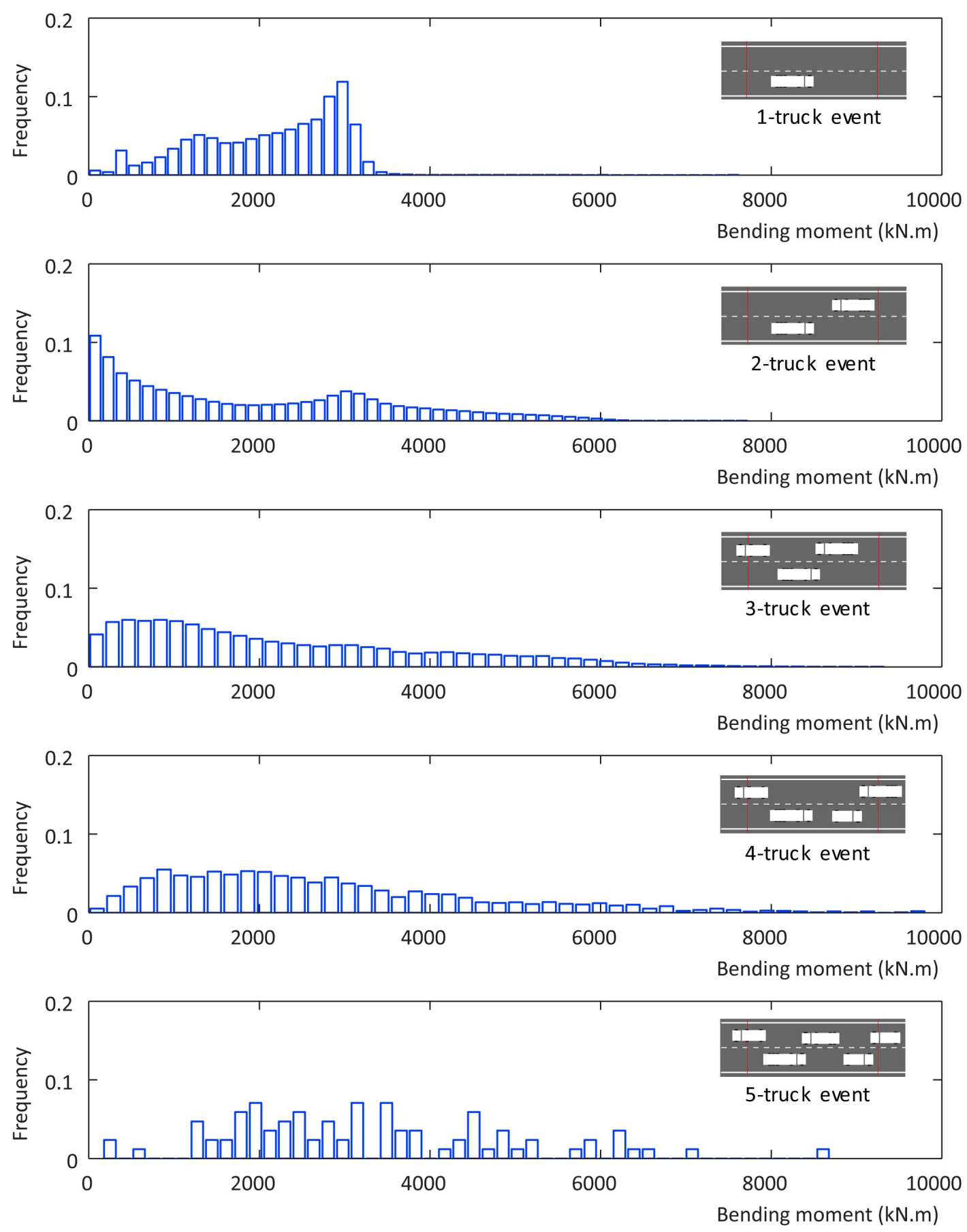

FIG. 5: Histogram of load effects due to various types of loading events 


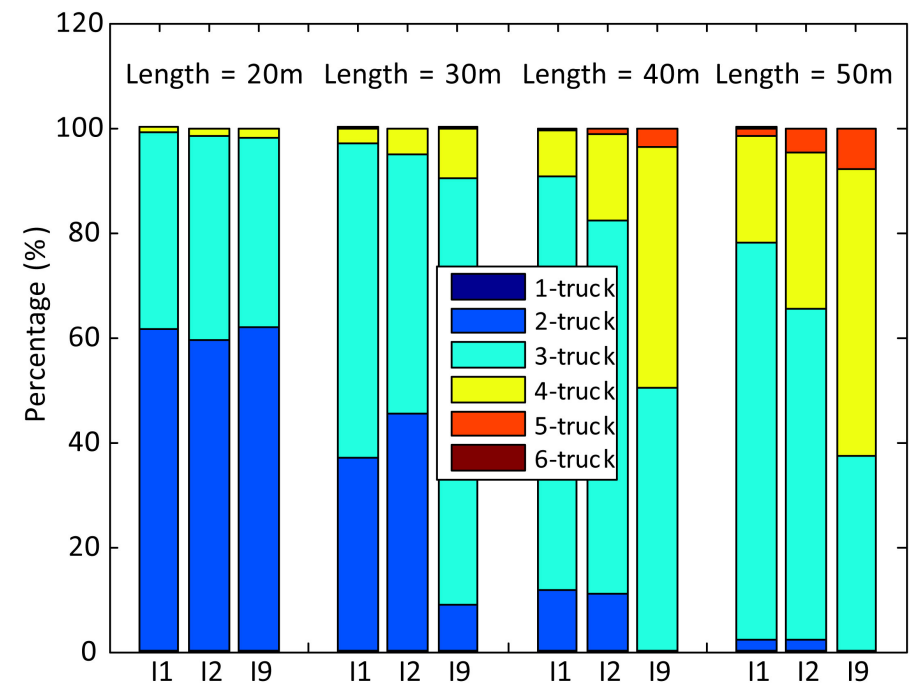

FIG. 6: Probabilities for six types of loading events (left) over $90^{\text {th }}$ percentile

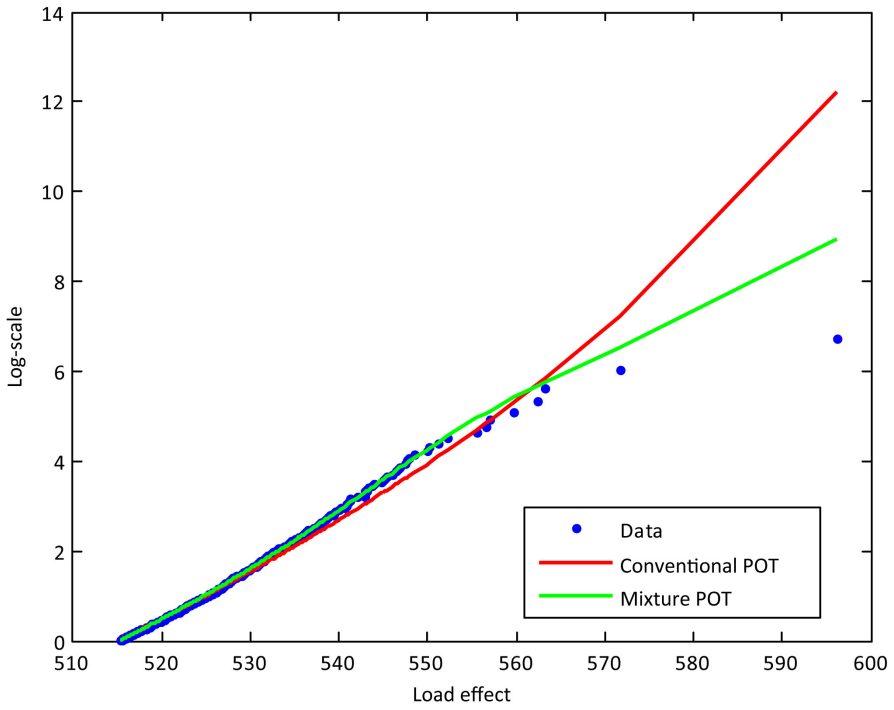

FIG. 7: Gumbel scaled cumulative distribution probability plot. 


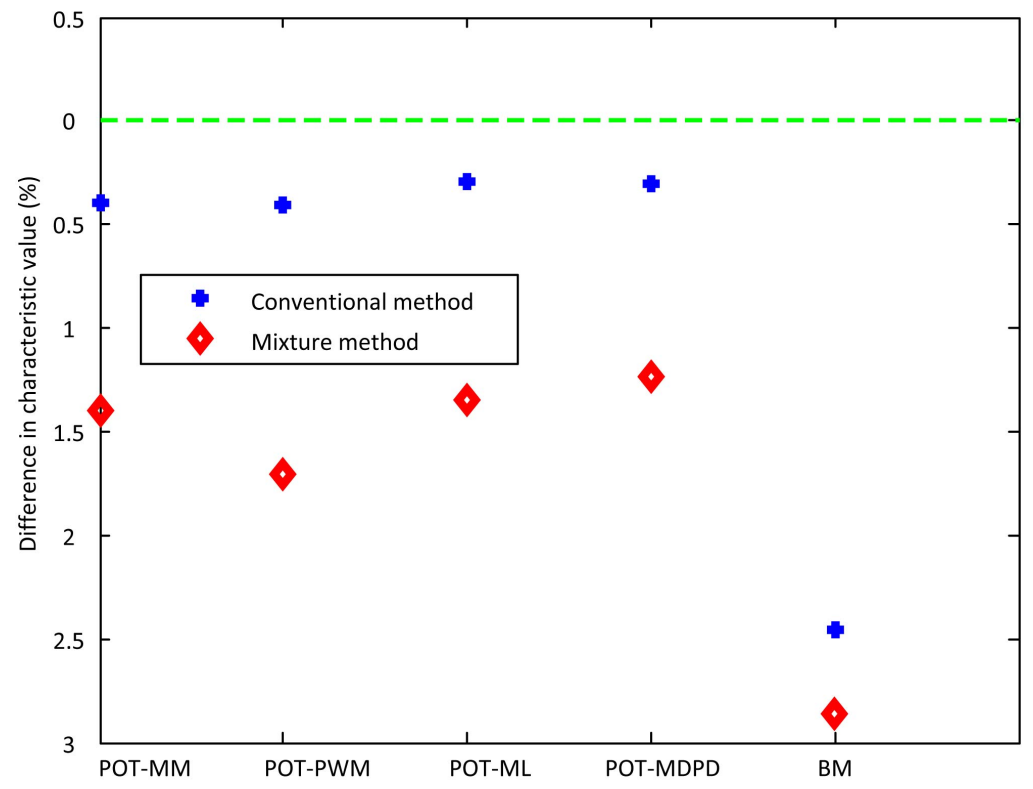

FIG. 8: Comparison of estimates of the characteristic values obtained from between CPOT and MPOT.

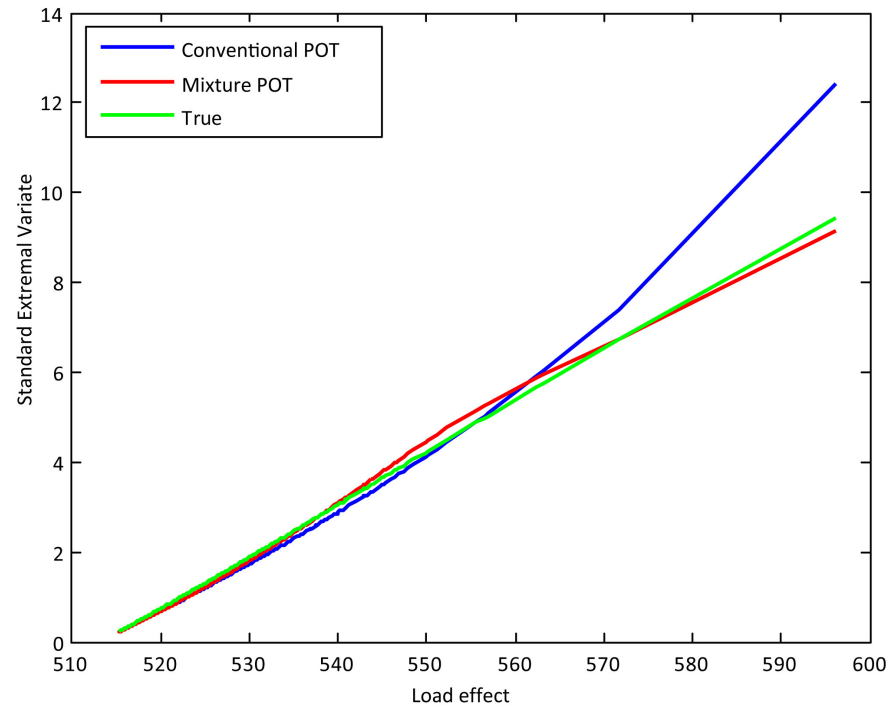

FIG. 9: Extreme value distribution from CPOT and MPOT with true distribution. 

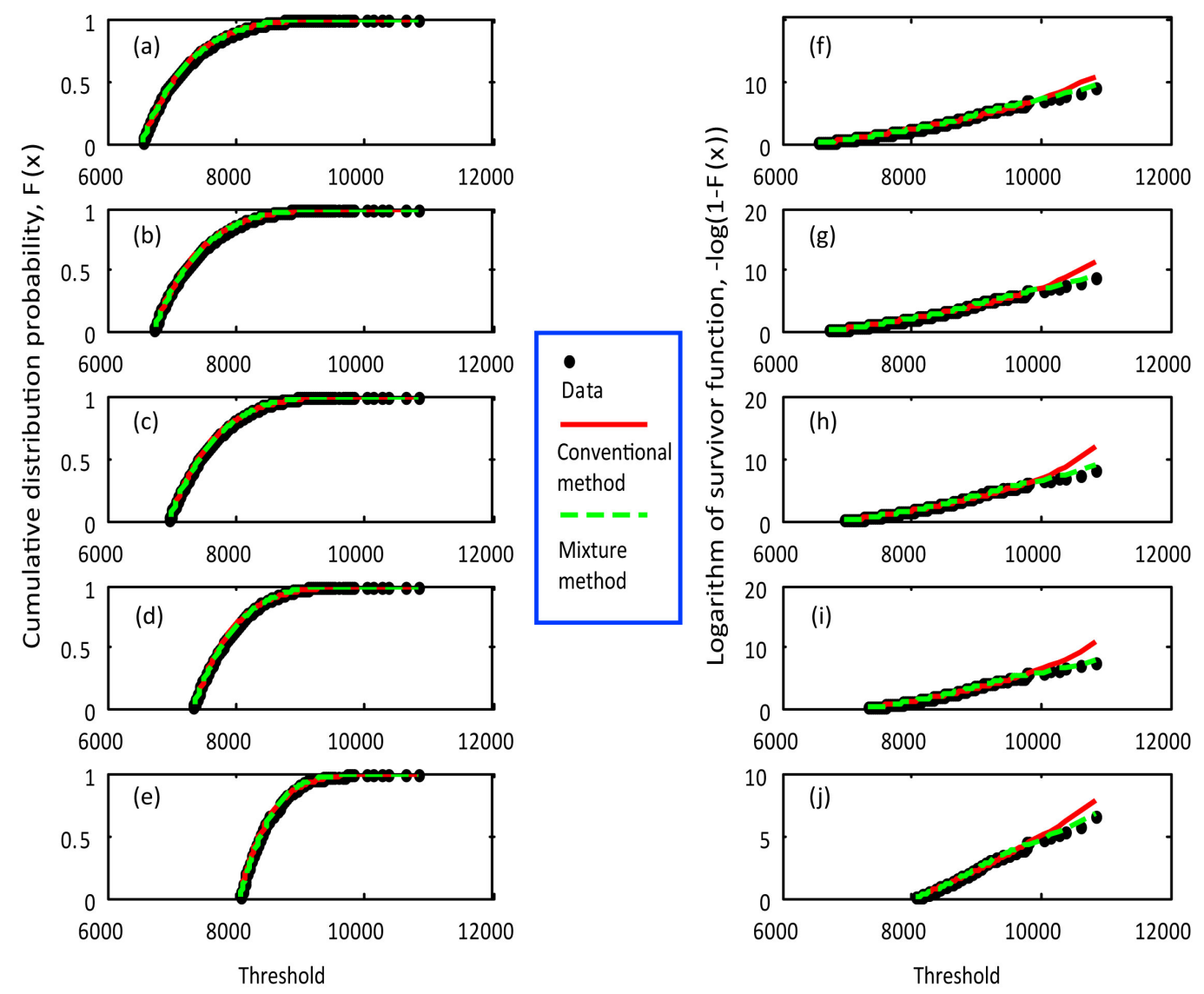

FIG. 10: Diagnosis plot for threshold excess model fitted to load effect. 